\title{
Interrelation Between Failure and Damage Accumulation in the Pre-Fracture Zone Under Low-Cycle Loading
}

\author{
Vladimir Kornev, Evgeniy Karpov and Alexander Demeshkin \\ Lavrentyev Institute of Hydrodynamics SB RAS \\ Russia
}

\section{Introduction}

When structures are loaded in catastrophic mode of operation, localization of irreversible strains occurs in regions of stress concentration. This is caused by the geometry of a structure or by the presence of fetaures (dints, holes, cracks, inclusions with mechanical characteristics different from properties of original material). If loading is in progressing, repeated loads cause gradual degradation of the material in regions of the localization of inelastic strain. As a result, this leads to generation and extension of cracks and to loss of a load capacity of the structure. Study of regularities of material degradation in regions of strain localization will permit one to appreciate possible structure resources in a catastrophic situation or consequence of failure, which affects subsequent behaviour of a structure in a common regime.

The distinctive features of fracture surface microrelief of metallic components in fatigue are fatigue striations oriented normally to the crack extension direction. It is appropriate to relate formation of fatigue striations to stepwise crack tip advance, and to record residual deflection of a beam under three-point bending when the loading corresponds to the lowcycle fatigue. A current striation may be formed after several loading cycles due to arrest of a fatigue crack after each advance of its tip in the Laird- Smith model (Laird \& Smith, 1962), the material being embrittled in a pre-fracture zone at each loading type. Damage accumulation in the pre-fracture zone is associated with accumulation of inelastic strains in this zone.

The aim of the present work was to study damage accumulation in the regions of inelastic strains near the notch tip having a finite width. Two cases are considered: i) symmetric three-point bending of a beam (the edge notch is made on the underside of a specimen in the transverse symmetry plane, damage accumulation is estimeted by the increment of a residual deflection); ii) tension of a plain specimen with a narrow edge notch (direct viewing of fatigue crack propogation was performed using digitized microscope with resolution of about $22500 \mathrm{pxel} / \mathrm{mm}^{2}$ ). Mechanisms of deformation, damage accumulation and failure of material under fatigue conditions have been proposed.

In the first case, the choice of geometry of a specimen and the loading type are governed by the following considerations. 
Under symmetric three-point bending of a beam with the transverse notch, main inelastic strains are concentrated ahead of the notch tip where stress concentration occurs. Change of mechanical characteristics of material in this region under repeated loading conditions causes the increase in residual deflection of the specimen. Thus, the amplitude of the residual deflection can be used as a measure of damage accumulation in the zone of localization of inelastic strains. This provides, from macroscopic phenomena, a possibility for qualitative and quantitative estimating the changes directly exhibited by the material due to processes of fatigue fracture. Moreover, in this case, the notch can be considered as a model of an edge crack with the blunted tip, and the region of localization of inelastic strains can be considered as a pre-fracture zone ahead of the tip of this crack.

In the second case, consider a plain specimen with one edge notch since two symmetrical notches lead to uncertainty in a choice of the point of crack initiation. Besides, after a crack initiates, the symmetry of a specimen is broken in one of paired notches and its initial symmetry loses significance.

\section{Low-cycle symmetric three-point bending of a beam with edge notch}

In the performed tests, the effect of various loading conditions, change in the geometry of a specimen, and preliminary plastic strain of material from which the specimens were made on the process of damage accumulation were studied. The attempt was made to determine parameters, which may be extended from the particular cases considered in the tests, to more general loading conditions. A possibility for description of regularities of damage accumulations with the aid of simple analytical functions involving constants just as determined from experiments, so specific for every material is considered.

The specimens were made from aluminum alloy D16T in the original state, so from preliminary stretched materials with the various degrees of plastic strain. The composition of the D16T alloy is as follows: $\mathrm{Al}$ was as a base metal, the alloying elements were $\mathrm{Fe}(0.3 \%)$, Si $(0,19 \%), \operatorname{Mn}(0.76 \%), \mathrm{Cu}(4.0 \%), \mathrm{Mg}(1.29 \%)$.

The experiments were conducted on electromechanical testing machine (the rated capacity load was $100 \mathrm{kN}$ ). The loading was repeated three-point bending with unloading and it was given by travelling of a moveable cross-head with a constant velocity. Loading diagrams were recorded at each loading cycle.

The minimum force of a cycle was $P_{\min } \approx 0$ for all the conducted tests. The maximum force of a cycle $P_{\max }$ was considered in three forms: $\left.i\right) P_{\max }=$ const (stationary low-cycle loading), ii) $P_{\max } \equiv P_{\max }(N)$ was increasing step-function of $N$ for which the number of cycles at one step was constant (non-stationary low-cycle loading with increasing load), and iii) $P_{\max } \equiv P_{\max }(N)$ was analogous decreasing step-function. The $P_{\max }$ value in all tests was chosen to provide plastic material deformation ahead of the notch tip. Tests under stationary cyclic loadings were conducted for different $P_{\max }$ values in the range from the limit of elasticity to the strength limit of a specimen. Besides, tests with different notch depth to beam height ratios were conducted.

Increment of the residual deflection $\delta w=\delta w(N)$ depends on the cycle number. The increment of the $\delta w$ deflection arises mainly due to mechanical properties of material in a pre-fracture zone near the notch tip, and therefore, as it was said before, it is considered as a measure of damage increment in this zone. The increment is considered to achieve the limit $w^{*}$ value if subsequent deflection of the beam proceeds without increase in $P$. 


\subsection{Stationary loading}

Fig. 1 demonstrates, as an example, the experimental diagram represented by curves of beam deflection $w$ versus applied force $P$ in stationary low-cycle testing a specimen made from D16T. The $P$ value is sufficiently large in order that fracture to happen after the limited number of cycles (in this case, $N^{*}=390$ ). This allows one to visualize all distinctive features of such diagrams obtained also for test materials for different $P_{\max }$ values. Curve 1 corresponds to single loading of a specimen until fracture occurs; group of curves 2 corresponds to cyclic loading up to the instant when a crack starts to extend for $P<P_{\max }$. Each curve of group 2 corresponds to loading branch of one cycle. All the curves of group 2, except the first one, have the initial horizontal section $P=0$. The length of this section is equal to the value of residual deflection accumulated at previous cycles. In this figure, $\delta w$ is the distance between adjacent curves of group 2 .

Damage accumulation can be divided into two specific stages. The first stage (Fig. 1, subgroup $A$ of curves 2) is a stage of cyclic strengthening at which decrease in $\delta w$ is observed with increase of $N$. At this stage, $\delta w$ achieves some minimum that is characteristic for the given value of $P_{\max }$ after which the $\delta w$ value becomes constant within the limits of measurement accuracy. The second stage (Fig.1, sub-group $B$ of curves 2 ) is characterized by the increase in the $\delta w$ value as $N$ increases, and this stage is accomplished by growth of a crack when $P<P_{\max }$. Accumulation of micro-defects during the first stage is likely to lead to formation of macroscopic defect, which then progresses during the second stage. Therefore, we call the second stage as a stage of development of a macro-defect. The ratio between the number of cycles in sub-groups $A$ and $B$ and the law of $\delta w$ variation depend on material characteristics (Karpov, 2009; Kornev et al., 2010).

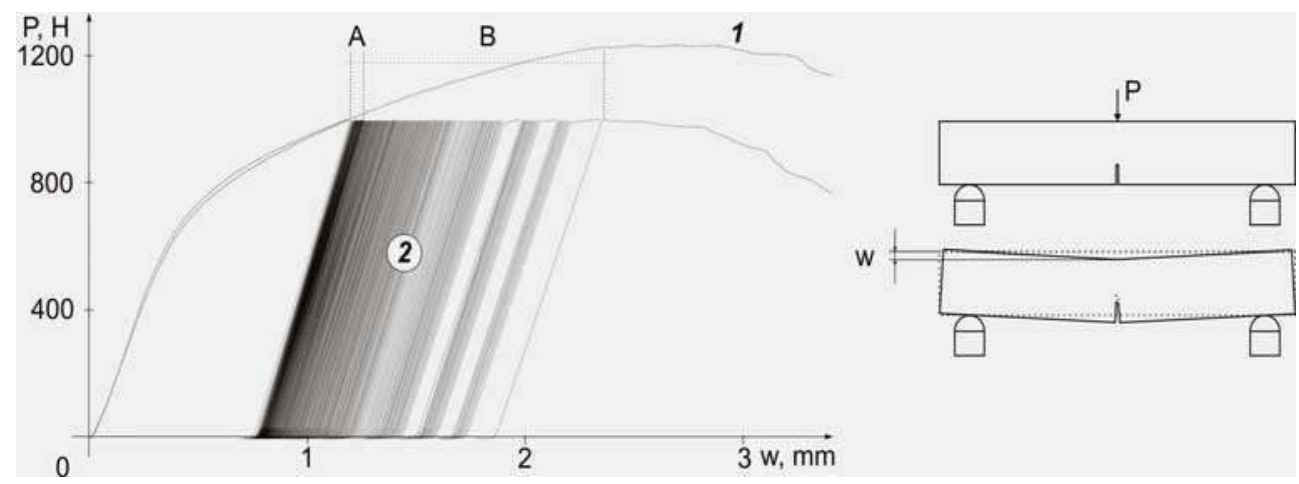

Fig. 1. Scheme of low-cycle test of specimen loaded in tree-point bending and plots of beam deflection as a function of force for every loading cycle for D16T alloy

Regularity in the residual deflection can be visualized as $\delta w(N)$ diagrams. An example of such diagrams is given in Fig. 2. Here pairs of curves are shown with numerals 1-5 for curves, one of the pairs being given by the analytical function and the second one being a saw-like profile. Here the saw-like profiles represent experimental $\delta w(N)$ curves for the D16T alloy. The analysis of experimental diagrams shows that curves can be approximated by plots of some power functions. These functions are to have asymptotes corresponding to the limits beyond which the process described by the diagrams can not take place. That is, the inverse power function with some scaling coefficient can be taken as approximating one. 
Besides, in the general case, both descending and ascending branches of the experimental curves should be approximated by different curves.

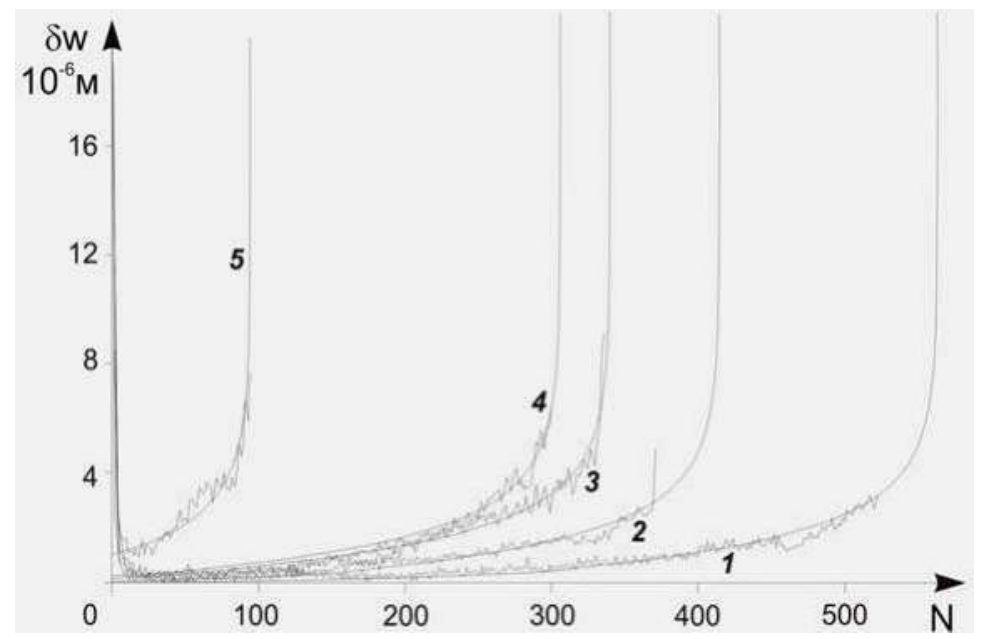

Fig. 2. Approximation of experimental curves of residual deflection increment for D16T alloy

The connection point of these curves corresponds to the minimum $\delta w$ value. In this case, the form of curve, which approximately describes either descending or ascending branch, is unique for all values of $P_{\max }$. That is, the curves of approximating functions for all $P_{\max }$ values can be superposed by parallel shift. Fig. 2. shows such curves (branches of hyperbolas corresponding to saw-like profiles 1-5). These curves are given by functions of the form $f(N)=\lambda(\alpha+\beta N)^{-\gamma}$ normalized in such a way that the experimental minimum point $\delta w$ would be a connection point of two curves approximating both the descending and ascending branches of the saw-like profile $(\alpha, \beta, \gamma, \lambda$ are experimental constants). If approximating functions have been defined, then the derivatives of these functions calculated for integers of the variable $N$ can be used as a magnitude characterizing material damage. The stage of cyclic strengthening $A$ of duralumin, which differs from steels by essentially larger grain sizes, is limited to several cycles even for low loads (Kornev et al., 2010). As compared with the $B$ stage, the stage $A$ can be neglected.

\subsection{Non-stationary loading}

Under non-stationary loading conditions, the process of cyclic strengthening begins again each time when $P_{\max }$ increases, and only the last stage explicitly includes both $A$ and $B$ stages of damage accumulation. However, if instead of change in $\delta w$, the analogous change in $\sum \delta w(N)$ (summation is performed over numbers of all cycles at one loading step), the stages $A$ and $B$ are also evident as in the case of the stationary loading. Fig. 3 demonstrates the experimental diagrams represented by curves of $w$ versus $P$ for non-stationary cyclic loading of alloy D16T.

The non-stationary loading with decreasing $P_{\max }$ shows that the initial overload of material provokes consequences, which may affect fracture process when loading with the low $P_{\max }$ 
value is applied again. Given in Fig. 4 are curves of $w$ versus $P$ for cyclic loading of D16T duralumin when decrease in $P_{\max }$ is step-like. Here, after some initial $P_{\max }$ value, monotonic nonlinear increase of $\delta w(N)$ is continued even for significant decrease in the $P_{\max }$ value. Beginning from the fourth step, $\sum \delta w(N)$ also increases. As a result, in spite of decreasing load, the specimen rapidly losses its load capacity. The tests also show that the increase in $P_{\max }$ after several steps of such loading leads to significant inelastic strain with strengthening for values of the force $P$, which earlier were appropriate to the linear dependence of the deflection on the applied force. This fact evidences that repeated loadings of a material preliminary experienced overloading lead to significant material damage even this loading is not followed by noticeable increase in the residual strain.

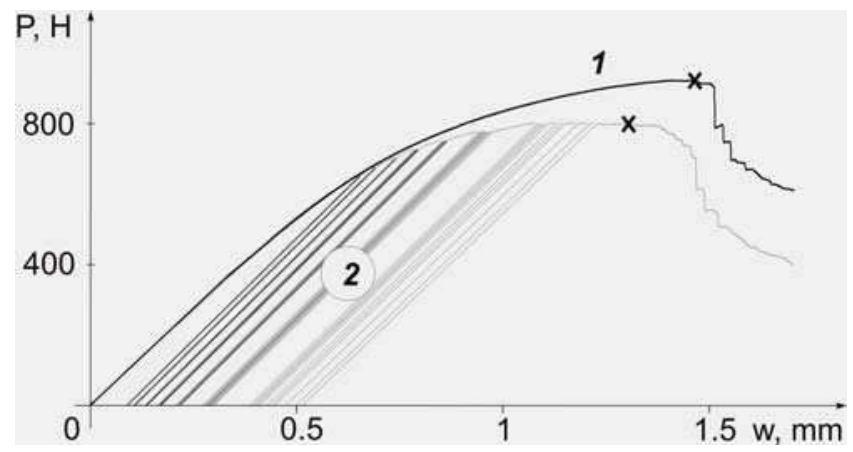

Fig. 3. Non-stationary cyclic loading with increasing maximal applied force for D16T alloy

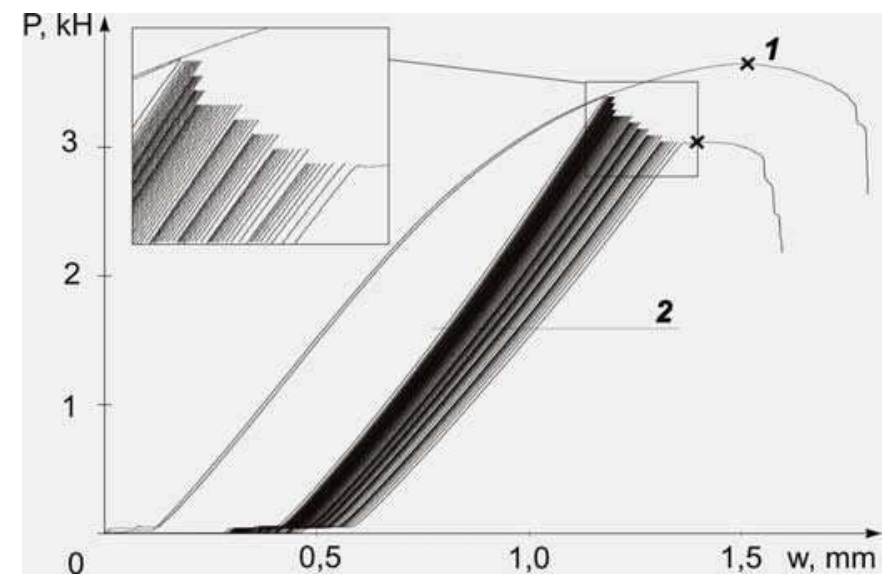

Fig. 4. Non-stationary cyclic loading with decreasing maximal applied force after initial overloading for D16T alloy

\subsection{The typical ratio}

The typical value for both stationary and non-stationary low-cycle loading is the ratio between limit deflections for single and repeated loading ( $w^{*}$ and $w^{* *}$, respectively). The 
tests show that this ratio for material is constant when different schemes of stationary and non-stationary loading are applied (Kornev et al., 2010). This allows one to use this ratio for comparison of results obtained on specimens with various geometrical dimensions and for different loading regimes. For duralumin, we have $w^{*} \geq w^{* *}$.

\subsection{Preliminary inelastic strain}

Preliminary inelastic strain of a material, from which the specimens have been made, essentially influences material resistance to cyclic fracture. As an example, Fig. 5 displays the experimental diagrams with curves of $w$ versus $P$ (Fig. $5(\mathrm{a})$ ) and the $\delta w(N)$ diagram (Fig. 5 (b)) for D16T duralumin with various degrees of preliminary stretching: diagram 1 for original materials, diagram 2 for materials stretched by 5\%, and diagram 3 for materials stretched by $10 \%$. All the specimens were loaded for the same $P_{\max }$ value, but in Fig. 5 (a), diagrams for three tests are displaced, for convenience, from each other along the horizontal axis.
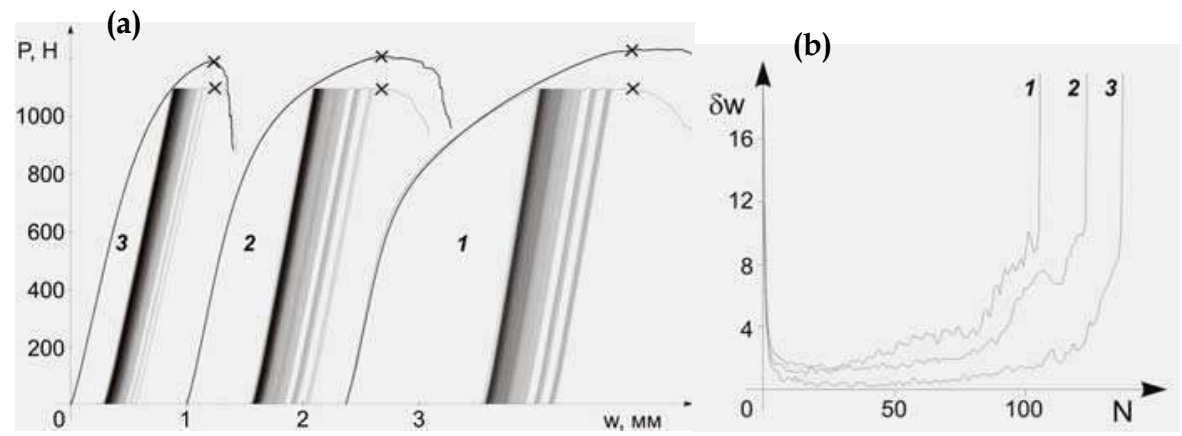

Fig. 5. (a), (b). Low-cycle loading of aluminum alloy after preliminary plastic deformation for D16T alloy

In Fig. 5(b), the area under the curve characterizes the limit deflection $w^{* *}$, which decreases as preliminary stretching increases. However, the decrease in $w^{* *}$ is followed by the decrease in $\delta w$. This leads to increase of the limiting number of loading cycles.

\subsection{Variation in the notch depth}

Comparison of tests conducted on beams with notches of different depths has shown that if $\delta w(N)$ diagrams have been plotted for some notch depth $l$, the diagrams can be used to obtain analogous diagrams for other $l$ values. Assume that $\delta w(N)$ diagrams have been plotted for the notch depth $l_{1}$, and for the notch depth $l_{2}$ there is the only diagram with curves of $w$ versus $P$ for single loading. The value of maximum applied force $P_{\max }=P_{1}$ corresponds to some deflection $w=w_{1}$ for $l_{1}$ under single loading, the limit deflection of cyclic loading for this notch depth being $w^{* *}=w_{1}^{* *}$, and the limit deflection of the single loading being $w^{*}=w_{1}^{*}$. In this case, the curve of damage accumulation $f_{1}(N)$ for $P_{\max }=P_{1}$, $l=l_{1}$ can be used to obtain the curve $f_{2}(N)$ for $P_{\max }=P_{2}, l=l_{2}$. Here $P_{2}$ is the force for which a specimen with the notch depth $l_{2}$ has the deflection $w=w_{2}$ such that

$$
\frac{w_{1}}{w_{1}^{* *}}=\frac{w_{2}}{w_{2}^{* *}}, \text { where } \frac{w_{2}^{* *}}{w_{2}^{*}}=\frac{w_{1}^{* *}}{w_{1}^{*}} \Rightarrow w_{2}^{* *}=\frac{w_{1}^{* *} w_{2}^{*}}{w_{1}^{*}} \text {. }
$$




\section{Development of fatigue crack under low-cycle tension conditions of a plain specimen with edge notch}

Tests on a plain specimen loaded in tension with the narrow edge notch were conducted. During the test, direct observation of fatigue crack propagation was performed using a digitized microscope with the resolution of $22500 \mathrm{pixel} / \mathrm{mm}^{2}$. The testing scheme is shown in Fig. 6. The field of view is outlined by dashed line.

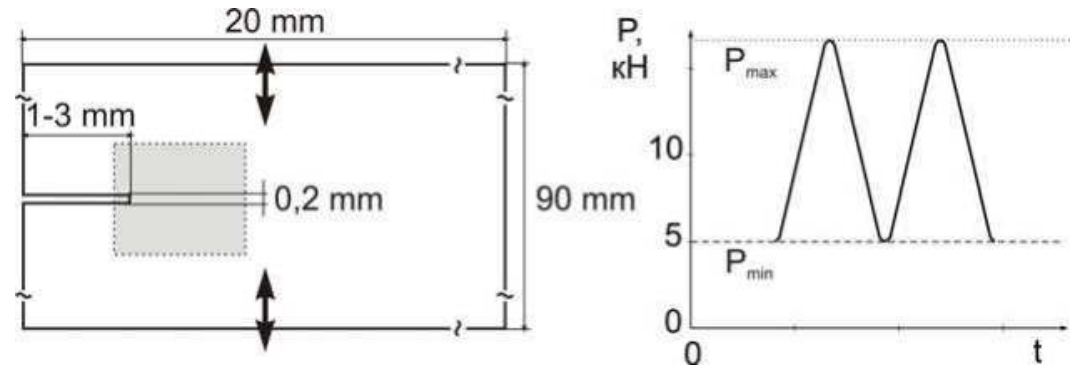

Fig. 6. Test scheme

The specimens were made from D16T alloy preliminary heat-treated at $500^{\circ} \mathrm{C}$ to give more plastic material. Plain specimens with notches of different lengths $(1-3 \mathrm{~mm})$ were used. The minimum load was the same in every cycle, the maximum load was chosen such that three different loading types were provided: $i$ ) near yield strength, ii) near the limit of load capacity, iii) the average value of them.

Photographs in Fig. 7 illustrate stages of crack propagation near crack-like defect in two cases $i$ ) continuous tension with constant rate, ii) low-cycle tension.

In the case of continuous tension, the following stages may be observed. First, intense plastic deformation ahead of the notch tip occurs and two zones of strain localization are formed with a delta-shaped area between these zones. The area we term as a pre-fracture zone since further it will define the crack extension direction. At this stage, several focuses of fatigue crack initiation are formed, which are located at notch angles as it is seen in the photograph (a.1). The pre-fracture zone is formed not by a prospective crack, but by the notch itself and its shape is unclear. Further (a.2), one of microcracks develops as a crack propagating within the zone of plastic strain localization irrespective of the pre-fracture zone specified by the notch. This zone becomes more structured and its tip is separated from the crack tip. Here the pre-fracture zone tip starts to shift towards the developing crack. At the next stage (a.3), crack branching takes place, the branches being formed just as near the crack, so at its faces. This evidences the significant extent of material embrittlement in the vicinity of crack extension. The angle at the pre-fracture zone tip starts to decrease. Then (a.4) the branch nearest to the pre-fracture zone tip has some advantages and defines the final direction of crack extension. When the crack tip joins the pre-fracture zone tip (a.5), the critical state is achieved after which the crack starts to extend very fast. The final failure of a specimen is preceded by a short stage (a.6), at which the angle at the pre-fracture zone crack becomes similar to the crack opening angle and one of pre-fracture zone edges defines a path of the subsequent crack extension unambiguously. The crack is very short before the critical state: its length is less than the notch width.

Under repeated low-cycle loading conditions, the pre-fracture zone created by a notch plays no noticeable role especially in the cases when cycle loading starts at insignificant plastic 


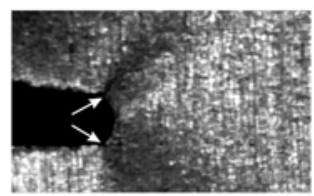

a.1

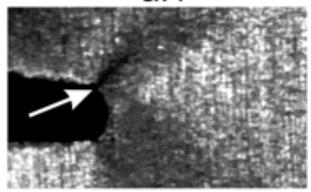

a.2

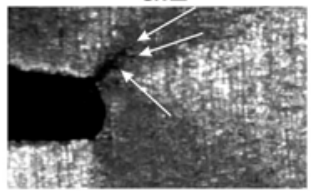

a.3

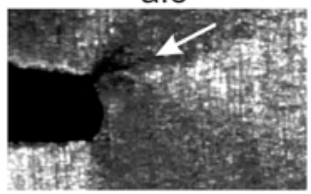

a.4

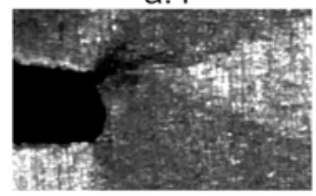

a.5

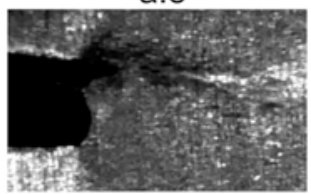

a.6

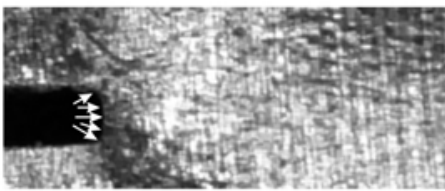

b. 1

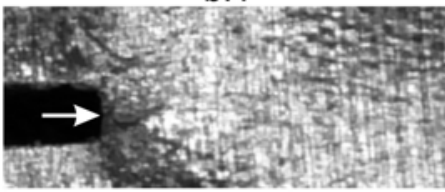

b. 2

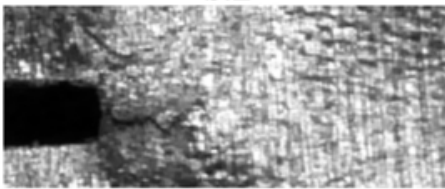

b. 3

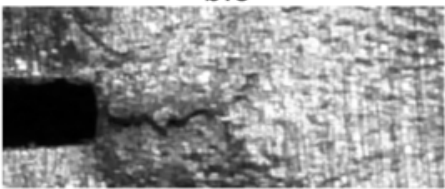

b.4

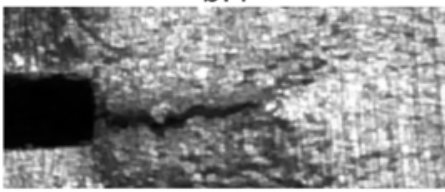

b. 5

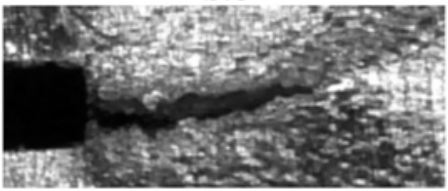

b.6

Fig. 7. Stages of crack propagation for D16T alloy; a.1. occurrence of incipient cracks under single loading conditions; b.1. the same under low-cycle loading conditions; a.2. growth of one of incipient cracks under single loading conditions; b.2. the same under low-cycle loading conditions; a.3. crack branching and moving of pre-fracture zone of a notch close together with the crack tip under single loading conditions; b.3. occurrence of "tooth" under low-cycle loading conditions; a.4. development of crack branch nearest to the pre-fracture zone of a notch under single loading conditions; b.4. growth of a crack beyond pre-fracture zone of a notch, formation of the proper pre-fracture zone for a crack under low-cycle loading conditions; a.5. merging pre-fracture zones of the crack and notch under single loading conditions; b.5. final stage of crack development under low-cycle loading conditions characterized by continuous growth of visible sizes of the pre-fracture zone and by decrease of the angle between whiskers; a.6. crack in critical state followed by fast final fracture under single loading conditions; b.6. the same under low-cycle loading conditions 
deformations of a specimen. In this case, zones of localization of maximum plastic strains at notch angles are slightly structured and the crack can start to develop from any point of the front bound of the notch (b.1). When one of microcracks starts to extend, a delta-shaped prefracture zone is formed ahead of the microcrack (b.2). When the crack is short, the length of the pre-fracture zone is defined by that of the edge notch. The angle between whiskers in the pre-fracture zone tip is close to the right angle, and visible sizes of this zone are of the order of surface roughness occurring due to plastic flow of material. Then, at some distance from the front edge of the notch, temporal crack arrest takes place in all the considered cases. Keeping constant length, the crack begins to open at the expense of blunting and increasing the length of its front edge, and then it changes both the direction and rate of propagation. The path, which the crack takes, deviates from the mean direction of its propagation and the former coincides with the upper or lower edge of the pre-fracture zone. After this, the crack returns to the mean path of propagation. The "tooth" formed at a crack face is clearly seen (b.3). Then the rate of crack growth is steadily increased, crack opening being continued. The pre-fracture zone length increases (b.4). The next to last stage is characterized by continuous growth of visible sizes of the pre-fracture zone and by decrease of the angle between whiskers (b.5). At last, the critical state is achieved (b.6): the crack is blunted, significant crack opening takes place that is comparable with the original notch width, then the crack produces a path branch sharply deviated from the mean path of propagation and then final failure of material occurs after which loading must be ceased. As opposed to continuous loading, the crack length to the instant of the critical state is close to the notch length.

Given in Fig. 8 are plots characterizing crack propagation as a function of the number of cycles. At the left, some geometric characteristics of cracks versus the number of cycles are given. At the right, $(P-\varepsilon)$ plots of specimens are given, where $\mathrm{P}$ is applied force, $\varepsilon$ is the overall elongation of a specimen. Both minimum and maximum loads of loading cycles are shown on these plots, as well as the maximum strain on the first loading cycle. The value of maximum force in a cycle was chosen such that to provide areas of plastic strain ahead the notch tip.

Plots a.1 and a.2, b.1. and b.2, and c.1. and c.2 correspond to specimens with the notch of 1 $\mathrm{mm}, 2 \mathrm{~mm}$ and $3 \mathrm{~mm}$ in length, respectively. Curves 1,2, 3 and 4 on the left plots correspond to the overall length of crack along its face, the distance of the crack tip from the front notch edge, the difference between these values, and the notch width increase of which shows opening of the crack mouth, respectively. All these values demonstrate nonlinear growth of cracks for which hyperbolic functions are applicable for description of both this growth and damage accumulation at the notch tip in the case of three-point bending of a beam. The general structure of material undoubtedly influences crack propagation as it can be seen on plots in Fig. 8 (a.1, b.1, and c.1), deviations of a crack due to structural heterogeneity being leveled out at its further propagation.

Fig. 9. illustrates separate parts of a crack corresponding to different stages of cyclic loading. Here a crack at the notch $3 \mathrm{~mm}$ in length is shown.

It seems likely that origination of a "tooth" is not a consequence of a heterogeneous structure of the material, but this is associated with sizes of defects from which a fatigue cracks start to propagate. In this case, a residual durability of the structure can be defined by its appearance.

Let us discuss interaction between plasticity zones formed in the vicinity of the notch tip and propagating cracks under single and cyclic loading conditions. Fig. 11 shows: a) schemes of zones of plasticity localization in the vicinity of the notch tip (region I) with 
features near angles (regions II and III), b) schemes of crack initiation under single loading conditions, c) scheme of crack initiation under cyclic loading conditions, d) scheme of fatigue crack propagation with origination of a "tooth" at the boundary of the zone of plasticity localization for a notch. Only in the case when load capacity is exhausted, initiation of cracks on the front notch edge at several points takes place: in original material under single loading conditions (Fig. 11.b), in material accumulated damages under fatigue loading (Fig. 11.c).

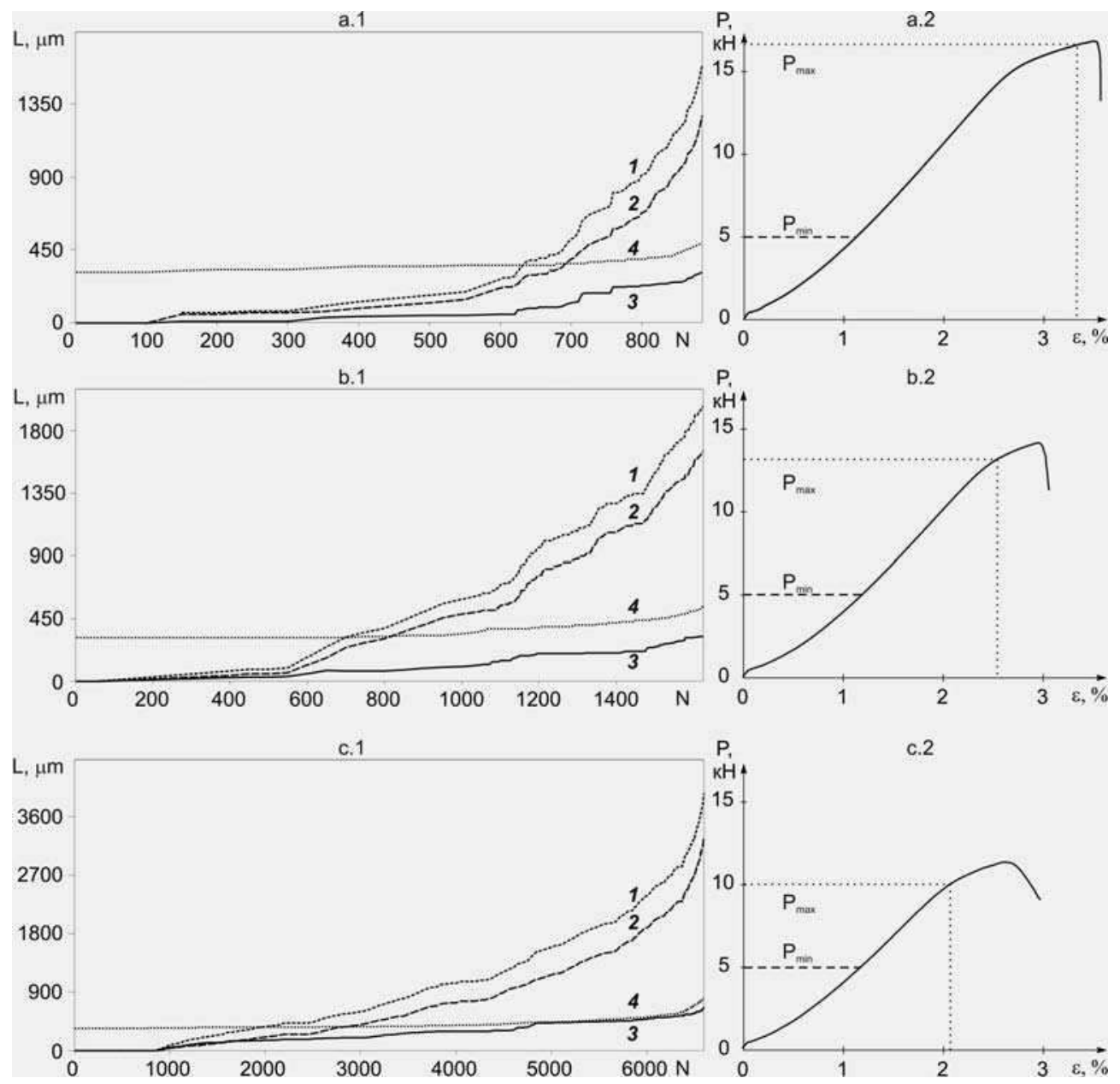

Fig. 8. Characteristics of fatigue crack propagation for D16T alloy

Given in Fig. 10 are forms of three cracks for notches of various lengths (notch lengths in $\mathrm{mm}$ are shown inside contours of final pre-fracture zones). Here it is seen that a "tooth" is inherent to all the cracks, the distances from the crack onsets to "teeth" are in direct proportion to lengths of notches. This "tooth" can be identified with pronounced fatigue striations originated on crack faces. In Fig. 10, all three "teeth" are marked with circles. The 
distance to a "tooth" is close to one third of the overall length of each crack. The segment on the horizontal axis in Fig. 8 is assigned to origination of a "tooth". This segment is nearly the same for all the cracks with respect to the total number of cycles. These segments correspond to about 600, 1100 and 4800 cycles, respectively, on a.1, b.1, c.1.

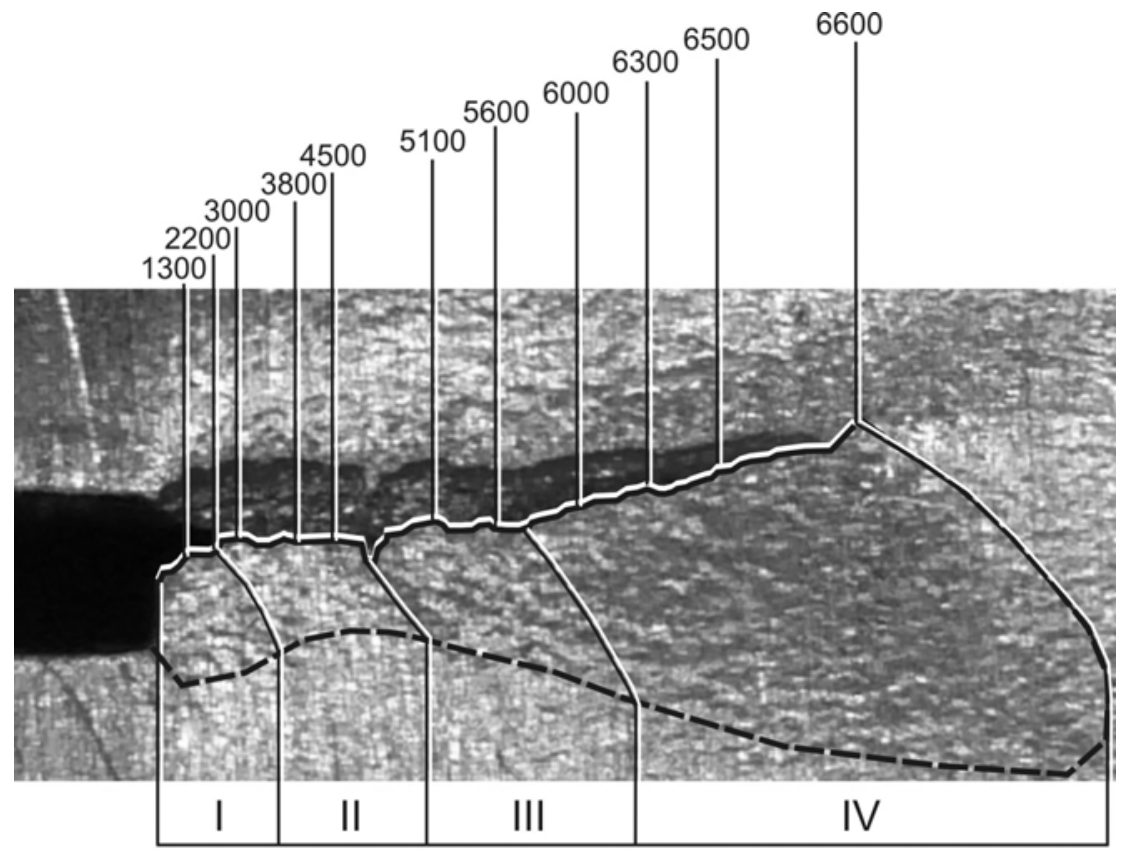

Fig. 9. Propagation of crack in pre-fracture zone for D16T alloy. Given at the top are the numbers of loading cycles, dashed line at the bottom outlines the visible area of maximum plastic strains. This area is divided into four regions: a crack initiates and propagates from the beginning in the closed state, and then it takes the mean propagation path (region I); the stage of stable crack propagation before a "tooth" arises (region II); stable crack propagation after "tooth" proceeds with increasing rate and continuous growth of the pre-fracture zone (region III); the final stage of crack propagations that is characterized on plots by clear nonlinearity (region IV)

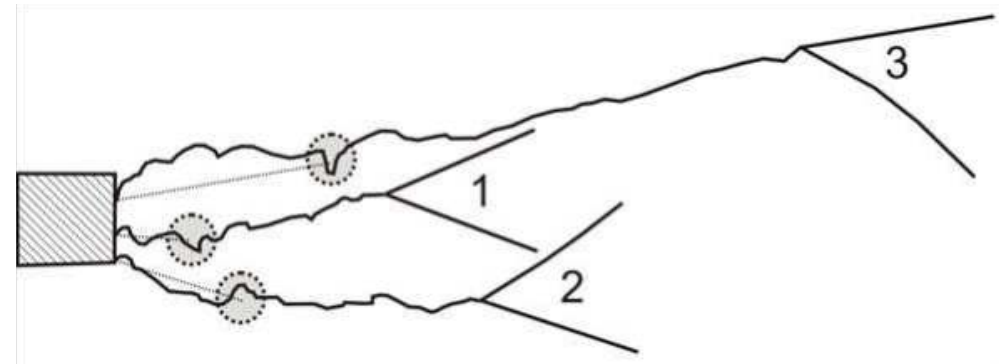

Fig. 10. Cracks and pre-fracture zones for various notch lengths 


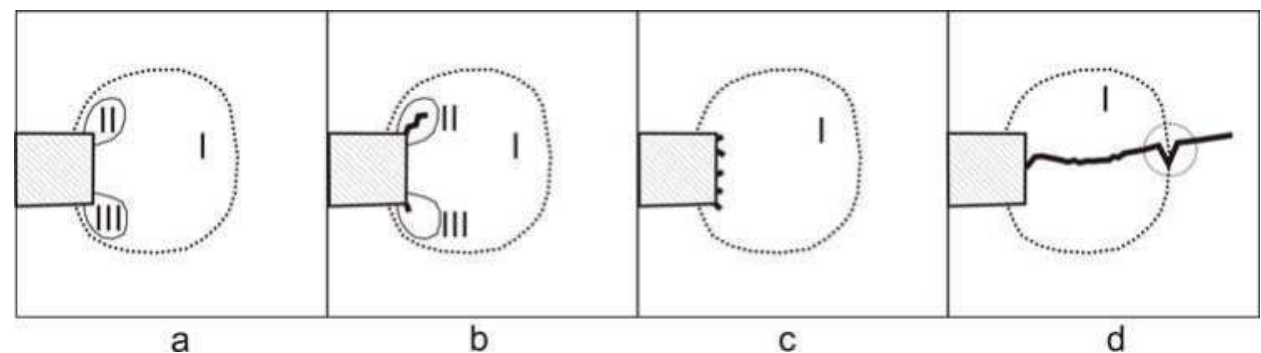

Fig. 11. Cracks and pre-fracture zones

At the initial stage, a fatigue crack passes through material located in the region of plasticity localization near the notch (region I in Fig 11.a, regions I and II in Fig. 9). Further, when the crack continues to propagate, the zone of plasticity localization ahead of the sharp crack tip serves as a pre-fracture zone. The former zone is sufficiently small. As a result of the fact that the crack tip passes from one region to another, a pronounced fatigue striation is originated in the form of a "tooth" (marked by circle in Fig 11.d).

\section{Comparison between test results and theoretical notions}

The model of low-cycle fatigue describing pulsing loading of a specimen with the inner macrocrack has been proposed in (Kornev, 2004, 2010). This model is appropriate to the scheme by Laird-Smith (Laird \& Smith, 1962; Laird, 1967). Within the framework of the proposed model in (Kornev, 2004, 2010), information on material strain in the pre-fracture zone has been obtained in detail: processes of damage accumulation, step-wise crack tip advance, and failure of structures for pulsing loading are described. Accumulation of damages is associated with inelastic strain of materials in the pre-fracture zone. The simple relations for the critical fracture parameters and the fatigue life have been obtained. Attention should be paid to the following circumstances: in the foregoing model, the information of damage accumulation and the hypothesis concerning the crack arrest are essentially used. Emphasize that when damages are accumulated, just as linear, so nonlinear summation of damage in the pre-fracture zone material may occur in the context of the considered model. In deciding between one and another way for summation of damages, no experimental data on damage accumulation at every loading step were available. The experimental data described in the previous section make up this deficiency.

The model in (Kornev, 2004, 2010) describes occurrence of striations under fatigue fracture. Material is considered to consist of quasi-brittle fibers separated by thin layers, which possess quasi-ductile fracture type before strain, and after inelastic strain of layers, the fracture type changes to quasi-brittle. The fiber diameters coincide with diameters of grains of tested materials (the diameters of grains are $\approx 10^{-2} \mathrm{~cm}$ ), and the fiber widths coincide with the thickness of layers separating subgrains. The width of the layers is $\approx 10^{-4} \mathrm{~cm}$. Further we assign numeral 1 to the fiber material and numeral 2 to the thin layer material. Properties of the layer material allow description of occurrence of some marks (whiskers or ears) from fatigue striations. In essence, in work (Kornev, 2004, 2010) there is considered the behavior of the simplest composite medium, material of which changes its fracture type under inelastic strain.

For deriving sufficient fracture criteria (Kornev, 2004, 2010) for low-cycle fatigue, modification of the classical Leonov-Panasyk-Dugdale model (Kornev, 2004) is used, where 
the pre-fracture zone is a rectangle ahead of the crack tip. The modification of the classical Leonov-Panasyk-Dugdale model allowed one to describe not only the pre-fracture zone length $\Delta_{1 \sigma+}$ at every loading cycle, but a magnitude of inelastic strain under tension $\varepsilon_{\sigma+}$ for material of the pre-fracture zone fiber nearest to the macrocrack center

$$
\begin{gathered}
\varepsilon_{\sigma+}=\frac{1-\frac{\sigma_{m 1}}{\sigma_{a}} \sqrt{\frac{r_{1}}{2 l}} \frac{k_{1}}{\sqrt{n_{1}}}}{\frac{5}{\pi(\eta+1)} \frac{G_{1}}{\sigma_{m 1}}}, \\
\frac{\Delta_{1 \sigma+}}{r_{1}}=\frac{k_{1}^{2}}{2 n_{1}} \frac{\left(\frac{5 \sqrt{2}}{4(\eta+1)} \frac{G_{1}}{\sigma_{m 1}} \varepsilon_{\sigma+}\right)^{2}}{\left(1-\frac{5}{\pi(\eta+1)} \frac{G_{1}}{\sigma_{m 1}} \varepsilon_{\sigma+}\right)^{2}}
\end{gathered}
$$

Here $\sigma_{a}$ is the amplitude of pulse loading; $\sigma_{m 1}$ is the limit of elasticity; $n_{1}$ and $k_{1}$ are integers $\left(n_{1} \geq k_{1}, k_{1}\right.$ is the number of damage-free material fibers); $n_{1} r_{1}$ is the averaging interval for the first material; $r_{1}$ is the specific linear dimension of the first material structure; $2 l_{0}$ and $2 l=2 l_{0}+2 \Delta_{1 \sigma+}$ are lengths of initial and fictitious cracks, respectively; $G_{1}$ is the shear modulus of fibers; $\eta=3-4 \mu$ and $\eta=(3-\mu) /(1+\mu)$ are coefficients for plane strain and plane stress state, respectively, where $\mu$ is the Poisson ratio; for relations (1), the restriction $1-5 G \varepsilon_{\sigma+} /\left(\pi \sigma_{m 1}(\eta+1)\right)>0$ holds.

Under cyclic pulse loading conditions, when the scheme of three-point bending is used, hysteresis loops take the form given in Fig. 12.

These hysteresis loops with translation differ from the standard statement in the model in (Kornev, 2004, 2010), in which the scheme of rigid loading under unloading is accepted. In Fig. $12, \varepsilon_{1}^{(i)}$ is the limit elongation of original materials for $i=0$; that after the first inelastic strain is $i=1$; that after the second inelastic strain is $i=2$, and etc. First three loops are depicted with lines widen from one loop to another, and the onset of the fourth loop is depicted with dots.

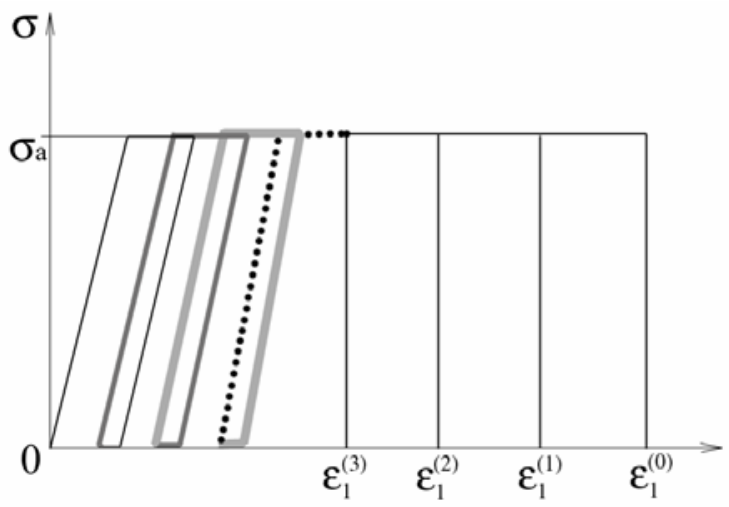

Fig. 12. Scheme of material damage 
Consider a typical situation. Let failure occur at the fourth loading cycle. During the strain process, the material elongations at the real crack tip coincide with the limit material elongation $\varepsilon_{1}^{(3)}$ after the third inelastic strain of the fiber nearest to the real crack tip (Fig. 12), and the crack tip being advanced.

Recall that the crack length in the model in (Kornev, 2004, 2010) changes by the pre-fracture zone length $\Delta_{1 \sigma+}$ after the step-wise real crack tip advance, and under the repeated loading, materials in the pre-fracture zone become brittle (Romaniv et al., 1990; Laird \& Smith, 1962; Kornev, 2004, 2010). Each such advance is associated with the certain number of cycles when linear and non-linear damages are summed. The performed tests show that the initial state of material, which falls into the pre-fracture zone, influences the process of the step-wise crack tip advance. The magnitude of inelastic strain under stretching $\varepsilon_{\sigma+}$ and the prefracture zone length $\Delta_{1 \sigma+}$ depend on: $i$ ) load amplitude $\sigma_{a}$; ii) initial crack length $2 l$; and iii) preliminary inelastic strain of material. If the basic parameter of inelastic strain $\varepsilon_{\sigma+}$ slightly depends on $2 l$, then for the pre-fracture length $\Delta_{1 \sigma+}$, the analogous dependence is pronounced. This corresponds to the passage of the second fracture process to the third one on the Paris curve (Romaniv et al., 1990; Shaniavski, 2003).

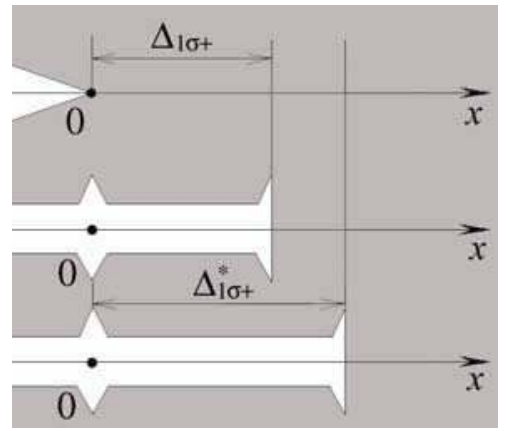

Fig. 13. Schematic drawing of material damage

As explained the step-wise advance of the real crack tip by the length $\Delta_{1 \sigma+}^{*}=\chi \Delta_{1 \sigma+}$, where $\chi$ is such a coefficient that for quasi-ductile layers $\chi=1$, and for quasi-brittle layers $\chi>1$.

The most important question is as follows: what is a distance from the crack onset where the crack will arrest? In the model in (Kornev, 2004, 2010), the case of original material loading is considered when the layers possess the quasi-ductile fracture type. Because of this, the ratio $\chi=1$ holds. After preliminary inelastic strain of a material from which specimens have been made, embrittlement of layer materials occurs, and the ratio $\chi>1$ can hold. The step-wise crack tip advance in composite materials with quasi-ductile and embrittled layers elucidates the schematic drawing in Fig. 13. At the scheme top, the right-hand tip of a blunted crack is shown before its start. In the middle part of the scheme, the crack tip advances by the segment $\Delta_{1 \sigma+}$, then the crack is blunted at the mesoscale, see the ratio $\chi=1$. At the scheme bottom, the crack tip advances by the segment $\chi \Delta_{1 \sigma+}$, and then the crack is blunted at the mesoscale, see the ratio $\chi>1$.

In essence, just as in the model in (Kornev, 2004, 2010), so in foregoing experimental results, the distinct influence of the single parameter on the process of damage accumulation under cyclic pulse loading is traced. In the model in (Kornev, 2004, 2010), it is the parameter that characterizes inelastic strain of material in the pre-fracture zone $\varepsilon_{\sigma+}$, and in experiments, it 
is the parameter, describing inelastic (residual) deflection of beams. In experiments, the residual deflection of beams characterizes both advance of the real crack tip and development of the pre-fracture zone after each loading cycle. The experimental results obtained agree with the diagram of fatigue failure with various kinds of relief elements formed as a result of fatigue fracture seen in Fig. 3.23 from (Shaniavski, 2003), just as the theory described in (Kornev, 2004, 2010), so the experimental data being correlated with the second and third stages of the Paris curve.

The model in (Kornev, 2004, 2010) gives no preference to linear or nonlinear summation of damages in metals (Romaniv et al., 1990; Coffin \& Schenectady, 1954) under cyclic loading. The performed tests show that damage accumulation, in general, is always nonlinear, however, if the loading is performed for $P_{\max }$ near to the limit of the specimen plasticity, there exists, as a rule, the large interval on the axis of the number of cycles $N$, where the $\delta w$ value is close to its minimum for given $P_{\max }$ value. Within this interval, the damage accumulation may be considered to be linear as it is under non-stationary loading with $P_{\max }$ values close to the limit of plasticity. In both cases, the model proposed in (Kornev, 2004, 2010) and the obtained experimental results agree well. In this case, the hypothesis concerning a crack arrest holds (Kornev, 2004, 2010): a crack arrests at the interface between a fiber and a layer and then it is blunted. This layer is the first one located beyond a pre-fracture zone $\Delta_{1 \sigma^{+}}$, and the zone itself is located in material not subjected to some plastic strain.

When a loading regime with increased $P_{\max }$ is considered (this regime models catastrophic overload of a structure with crack), the model like that in (Kornev, 2004, 2010) is proposed, and the experimental results obtained agree essentially worse. Such disagreement of theoretical notions (Kornev, 2004, 2010) with the experimental results is explained by the relations $\chi=1$ or $\chi>1$. After the first catastrophic series of loadings, the intensity of damage accumulation process in material significantly increases. In this loading regime, the refined hypothesis $\chi>1 \quad \chi>1$ concerning crack arrest holds: a crack arrests at the fiberlayer interface at the distance $\Delta_{1 \sigma+}^{*}>\Delta_{1 \sigma+}$ and it is blunted, this interlayer is located beyond the pre-fracture zone $\Delta_{1 \sigma+}$, and the last is in material that has already been subjected to some plastic strain and embrittlement (Romaniv et al., 1990; Laird \& Smith, 1962; Kornev, 2004, 2010).

\section{Conclusion}

Recording the loading diagrams for beams with the edge crack allows description of both damage accumulation at the macroscale and failure of constructions at every loading cycle. The damage accumulation in the pre-fracture zone is associated with the residual deflection of beams after unloading.

Preliminary plastic deformation of aluminum alloy leads to decrease in its durability. However, here the intensity of damage accumulation also reduces under low-cycle threepoint bending of beams. This results in increase in the number of loads, which structure may withstand.

In the chapter, loading regimes with gradual overloading and with increased loading have been considered. These regimes model a common situation and catastrophic overloading under cyclic loading. The results obtained may be useful for prediction of fatigue life of a structure with the crack and in analyzing situations after overloading.

Direct observation in the vicinity of the notch tip during the process of low-cycle tension of a plain specimen allows one to trace the behavior of a fatigue crack at various stages of its 
propagation: i) crack initiation in the vicinity of stress concentration; ii) crack propagation within the plasticity zone; iii) onset of generation of a narrow pre-fracture zone formed by the crack itself; iiii) fast crack propagation beyond the plasticity zone.

Schemes allowing description of deformation, damage accumulation, and failure of material under fatigue with account of the preliminary inelastic deformation of the material and effect of stress concentration on crack initiation have been proposed.

\section{Acknowledgment}

The work was financially supported by Russian Foundation for Basic research (No 08-0100220), in the context of the project No 23.16 included into the program of Presidium of Russian Academy of Sciences, and of Integration Project of SB RAS, UB RAS, FEB RAS No 119.

\section{References}

Romaniv, O.N.; Yarema, S.Ya.; Nikiforchin, G.N.; Makhutov, N.A.; Stadnik, M.M. (1990). Fatigue and cyclic fracture toughness of structural materials, Vol. 4. Fracture mechanics and strength of materials, in four volumes, Naukova Dumka, ISBN 5-12-000489-X, Kiev, Russia (in Russian)

Shaniavski, A.A. (2003). Safety fatigue fracture of elements of aircraft constructions. Synergetic in engineering applications. Monografiya, ISBN 5-94920-015-2, Ufa, Russia (in Russian)

Laird, C.; Smith, G.C. Crack propagation in high stress fatigue. The Philosophical Magazine, A. Journal of Theor. Experim. and Aplied Physics, Vol.7. No.77, (1962), pp. 847-857, ISSN 1478-6435

Laird, C. The influence of metallurgical structure on the mechanism of fatigure crack propagation. Fatigue Crack Propagation, ASTM STP 415; Am. Soc. Testing Mats. (1967), pp. 131-168, ISBN 0-8031-1250-5

Kornev, V.M. Two-scale model of low-cycle fatigue. Change from quasi-ductile to brittle fracture. Strain and fracture of materials. No.2, (2008), pp. 2-11 (in Russian), ISSN 1814-4632

Kornev, V.M. Distribution of stresses and crack opening displacement in the pre-fracture zone (Neuber-Novozhilov approach). Physical Mesomechanics. Vol.7, No.32, (2004), pp. 53-62 (in Russian), ISSN 1683-805X

Nikitenko, A.F. (1997). Yield and long strength of metallurgical materials. Institute of Hydrodynamics, Siberian Branch of Russian Academy of Sciences, Novosibirsk, Russia (in Russian), ISBN 5-7795-0024-X

Coffin, L.F.; Schenectady, N.Y. A Study of the effects of cyclic thermal stresses on a ductile metal. Transactions of the ASME. Vol.76., No.6., (1954), pp. 931-950, ISSN 0742-4795

Karpov, E.V. Deformation and fracture of a spheroplast under low-cycle loading at various temperatures. Journal of Applied Mechanics and Technical Physics, Vol.50, No.1, (2009), pp. 163-169, ISSN 0869-5032

Kornev, V.M. Two-scale model of low-cycle fatigue. Embrittlement of pre-fracture zone material. Procedia Engineering. Vol.2, No.1, (2010), pp. 453-463, ISSN 1877-7058

Kornev, V.; Karpov, E.; Demeshkin, A. Damage accumulation in the pre-fracture zone under lowcyclic loading of specimens with the edge crack. Procedia Engineering. Vol.2, No.1, (2010), pp. 465-474, ISSN 1877-7058 


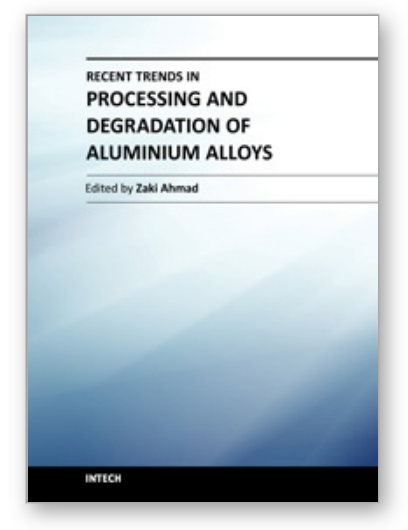

\author{
Recent Trends in Processing and Degradation of Aluminium Alloys \\ Edited by Prof. Zaki Ahmad
}

ISBN 978-953-307-734-5

Hard cover, 516 pages

Publisher InTech

Published online 21, November, 2011

Published in print edition November, 2011

In the recent decade a quantum leap has been made in production of aluminum alloys and new techniques of casting, forming, welding and surface modification have been evolved to improve the structural integrity of aluminum alloys. This book covers the essential need for the industrial and academic communities for update information. It would also be useful for entrepreneurs technocrats and all those interested in the production and the application of aluminum alloys and strategic structures. It would also help the instructors at senior and graduate level to support their text.

\title{
How to reference
}

In order to correctly reference this scholarly work, feel free to copy and paste the following:

Vladimir Kornev, Evgeniy Karpov and Alexander Demeshkin (2011). Interrelation Between Failure and Damage Accumulation in the Pre-Fracture Zone Under Low-Cycle Loading, Recent Trends in Processing and Degradation of Aluminium Alloys, Prof. Zaki Ahmad (Ed.), ISBN: 978-953-307-734-5, InTech, Available from: http://www.intechopen.com/books/recent-trends-in-processing-and-degradation-of-aluminium-

alloys/interrelation-between-failure-and-damage-accumulation-in-the-pre-fracture-zone-under-low-cycle-loadi

\section{INTECH}

open science | open minds

\section{InTech Europe}

University Campus STeP Ri Slavka Krautzeka 83/A 51000 Rijeka, Croatia Phone: +385 (51) 770447

Fax: +385 (51) 686166 www.intechopen.com

\section{InTech China}

Unit 405, Office Block, Hotel Equatorial Shanghai No.65, Yan An Road (West), Shanghai, 200040, China 中国上海市延安西路65号上海国际贵都大饭店办公楼 405 单元 Phone: +86-21-62489820

Fax: $+86-21-62489821$ 
(C) 2011 The Author(s). Licensee IntechOpen. This is an open access article distributed under the terms of the Creative Commons Attribution 3.0 License, which permits unrestricted use, distribution, and reproduction in any medium, provided the original work is properly cited. 